\title{
Interevent anticipation of liquid and solid sucrose rewards
}

\author{
RICHARD A. BURNS and LARRY P. WILEY \\ Georgia Southwestern College, Americus, Georgia
}

\begin{abstract}
Albino rats were trained in a straight runway under two different series of reward magnitude each day. In one three-run series (a trial), reward was given on the first and third runs (R-N-R), and in the other, reward was given only on the first run (R-N-N). The interval between trials was approximately $40 \mathrm{~min}$, and the interval between runs within a trial was approximately $30 \mathrm{sec}$. For one group of rats, reward was $1045-\mathrm{mg}$ sucrose pellets. For the other group, reward was $1 \mathrm{ml}$ of $30 \%$ liquid sucrose mixed by weight with tap water. With both reward types, running on the second run of a trial was faster if the third run was rewarded (R-N-R) than if it was not (R-N-N). This result suggests that the animals were in some way anticipating the reward amount for an upcoming run while running a current run. After 26 training days, the third run of the R-N-R trial was shifted to nonreward for 12 additional days. Times slowed on Runs 2 and 3 in the shifted trial, but there was no evidence of a successive negative contrast effect. The implications of the anticipation result for the interpretation of differences between sugar and ordinary food rewards in paradigms that suggest reward anticipation are discussed.
\end{abstract}

Whether anticipation is a necessary construct in the analysis of animal learning is, of course, an enduring concern. For some of those who imagine its necessity, attention has focused on possible mechanisms (e.g., Spence, 1956), yet others have chosen to abandon concern for mechanisms and to concentrate instead on functional properties. Characteristic of the latter strategy is a recent report that gives evidence of interevent anticipation. Rats given regular series of reward magnitude in a runway perform differently in some cases on comparably rewarded runs that precede differently rewarded runs (Capaldi, Nawrocki, \& Verry, 1983; Self \& Gaffan, 1983).

An old experiment (Elliott, 1928) that with its numerous contemporary replications has been the basis for most theoretical work on anticipation showed the successive negative contrast effect (SuNCE), a precipitous slowing in the running of rats given a reduced reward following regular experience with a larger reward. The SuNCE has long implied the operation of anticipation in that it has been viewed as the consequence of experiencing a lessthan-anticipated reward (Amsel, 1958). An impressive number of experiments, however, have failed to show the SuNCE when the reward is sugar (Flaherty, 1982). One vague possibility is that sugar somehow interferes with the mechanism of expectancy. For a preliminary look at this possibility, we have begun to study interevent anticipation with sugar rewards.

Rats were runway trained under two different series of reward magnitude each day. In one three-run series (a trial), reward was given on the first and third runs (R-N-R), and in the other, reward was given only on the

This work was funded by a grant to the first author from Charles L. Mix Foundation. Address correspondence to either author, Department of Psychology, Georgia Southwestern College, Americus, GA 31709. first run (R-N-N). For one group of rats, the reward was $1045-\mathrm{mg}$ sucrose pellets. For the other group, reward was $1 \mathrm{ml}$ of $30 \%$ liquid sucrose. Interevent anticipation in such a design would appear as faster running on Run 2 of trials that end with a rewarded run (R-N-R) than on Run 2 of trials that end with a nonrewarded run (R-N-N). Our thought was that if the mechanisms of anticipation underlying the SuNCE and interevent anticipation were the same, a reduction or elimination of the latter effect would be seen with sucrose.

\section{METHOD}

\section{Subjects}

The subjects were 12 naive Dublin Sprague-Dawley rats (male) approximately 90 days old at the beginning of preliminary training. The rats were obtained from Dominion Laboratories, Dublin, Virginia.

\section{Apparatus}

The apparatus was a wooden runway, $15.2 \mathrm{~cm}$ high, with a hinged clear plastic cover. The startbox $(20.3 \times 15.2 \mathrm{~cm})$ and alley $(182.9$ $\times 10.2 \mathrm{~cm})$ were painted black, and the goalbox $(30.5 \times 15.2 \mathrm{~cm})$ was painted white and was positioned at a $90^{\circ}$ angle to the end of the alley. Manually operated start and retrace doors were positioned at the beginning of the alley and $30.5 \mathrm{~cm}$ before the goalbox. Three photocells mounted 7.6, 160.0, and $195.6 \mathrm{~cm}$ beyond the start door allowed measurement of start, run, and goal times by Standard electric timers in units of $.01 \mathrm{sec}$.

\section{Preliminary Training}

All animals were housed in individual living cages in the room in which the experiment was conducted; water was freely available. During the first 8 days of preliminary training, the rats were reduced to $85 \%$ of their free-feeding body weights and allowed 15 min each day in squads of six on a walled exercise table. After random assignment to one of two groups, 2 days of table placements that consisted of 15-min free access to reward delivered on the exercise table in the goalcup (fashioned from a teaspoon) were administered. The reward for one group $(n=6)$ was a $30 \%$ sucrose solution mixed by weight in tap water. For the second group $(n=6)$, the reward was $45-\mathrm{mg}$ Noyes Formula F sucrose 
pellets. On the remaining 3 days of preliminary training, each rat was placed in the goalbox once each day with the retrace door closed and the goalcup baited with either $1 \mathrm{ml}$ of the liquid or 10 of the pellets, depending on the group assignment. By the end of goalbox placements, all rats were reliably consuming the reward in less than $40 \mathrm{sec}$. The order of running of the rats throughout the experiment was determined randomly each day, and the weighing and feeding of the animals took place about $1 \mathrm{~h}$ (spent on the table) after the completion of daily training.

\section{Training}

The design dictated that each rat would be given two three-run trials each day. In one of the two kinds of trial, the third run would be rewarded with the appropriate reward type (R-N-R), whereas in the other kind of trial, it would not (R-N-N). The first and second runs for both trials would be rewarded and not rewarded, respectively. Subgroups in which the order of trials within a training day was a fixed factor $(n=3)$ were formed pseudorandomly. The three runs of a trial were done in succession for each rat, and the second trial of a day was administered after every animal had completed the first trial (in the same order). The procedure was such that the interval between runs within a trial was about $30 \mathrm{sec}$ and the interval between trials was about $40 \mathrm{~min}$. The rats spent these intervals in the living cage. A run began with the opening of the start door $2 \mathrm{sec}$ after placement of the rat in the startbox and ended in the completion of the reward (judged by the experimenter) on rewarded runs or after $30 \mathrm{sec}$ in the unbaited goalbox on nonrewarded runs. The retrace door was closed as the rat passed the second photocell. Only one trial was given on each of the first 4 days of training; two of each kind of trial were given during this period in the appropriate order.

The procedure was a close approximation, with the exception of the reward types, to that of Capaldi et al. (1983, Experiment 1), in which interevent anticipation was demonstrated with food-pellet rewards. Because of our interest in successive contrast, however, we appended a 12-day phase to the 26 days of training with the primary procedure. During this phase, the R-N-R trial also had a nonrewarded third run, $\mathrm{R}-\mathrm{N}-\mathrm{R}(\mathrm{N})$.

\section{RESULTS AND DISCUSSION}

All times were logged, and separate factorial analyses combining the two levels of reward type and two levels of order of presentation of trials over runs, days, and trials were done on start, run, goal, and total measures. Because there were no important differences in the pattern of results for the different measures, the total measure is used here as the basis for discussion. Two animals, one of each reward type, were eventually discarded for repeated biting.

Plotted in Figure 1 are the mean transformed total times, $10[\ln ($ start) $+\ln ($ run $)+\ln$ (goal), during the 26 days of primary training. Each point is a daily mean for a particular trial averaged over reward types. Runs 1, 2, and 3 are depicted in the left, center, and right panels, respectively. Figure 1 shows the anticipation effect developing over days, with Run 2 times on R-N-R trials being comparable to those on Runs 1 and 3 . The Run 2 times on R-N-N trials are slower than those on Runs 1 and 3, although a trial discrimination that came from slowing on R-N-N trials did develop on Run 3. Statistical support for these conclusions is best summarized by a reliable runs $\times$ trials $\times$ days interaction $[F(50,300)=2.98, p<.01]$. Neither the interaction $(\mathrm{F}<1)$ nor any other experimental effects depended on reward type, and there were no effects involving the order variable that were not most reasonably described as warm-up effects.

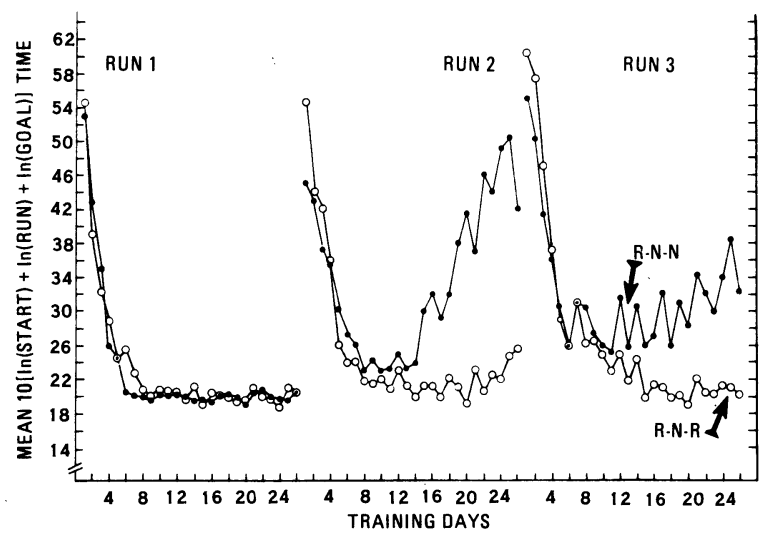

Figure 1. Transformed mean totals for the first 26 days of training averaged over the two reward types. The figure depicts the runs $x$ trials $\times$ days interaction.

Factorial analyses were performed separately on Runs 1,2 , and 3 to clarify the interaction. There was, of course, no evidence of differences in running for the two kinds of trial on Run $1[F(1,6)=1.39, p<.05]$, but the main effect of trials was reliable on Runs 2 and $3[F(1,6)=$ $71.78, \mathrm{p}<.01 ; \mathrm{F}(1,6)=52.65, \mathrm{p}<.01]$, as were the interactions of trials $\times$ days $[F(25,150)=7.24, p<.01$; $F(25,150)=3.28, p<.01]$. The Bonferonni t statistic with an error term constructed from the overall analysis was used to evaluate differences within a trial on given days across different runs, and it showed that times on Run 2 were slower than those on Run 3 in the R-N-N trial on Days 20 and 22-26 (k=12, $\alpha=.01)$. No other such comparisons were reliable for Runs 2 and 3.

The focus of this experiment does not warrant elaborate concern over factors that are responsible for the anticipation effect and slower running on Run 2 than on Run 3 in the R-N-N trial. Such a discussion can be found in Capaldi et al. (1983), Capaldi and Verry (1981), and Self and Gaffan (1983). What is of concern is the demonstration of what has been called interevent anticipation (Capaldi et al., 1983) with liquid and solid sucrose rewards, neither of which have produced evidence of anticipation's consequences in the negative contrast design (Burns, 1984; Burns, Lorig, Carr, \& Worthy, 1984). Since the SuNCE and interevent anticipation have both been shown with ordinary food pellets, but only interevent anticipation has been shown with sucrose, several possibilities regarding mechanisms are raised. If anticipation is at work in both phenomena, the mechanisms may be different, one functioning with both rewards, the other only with food. It may also be, of course, that one or both of the phenomena do not involve anticipation, or that the SuNCE involves the additional role of anticipatory frustration that is blocked in some way with sugar but not with food (Stanton \& Amsel, 1980).

In the second phase of this experiment, there was a slowing of times on R-N-R(N) trials in Runs 2 and 3, with no effect on Run 1. This phase is depicted in Figure 2, which is constructed in the same manner as Figure 1. 


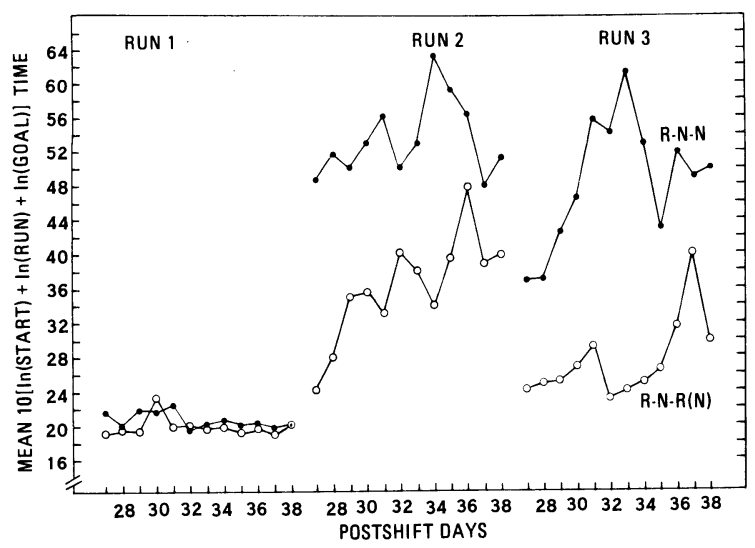

Figure 2. Transformed mean totals, shown in a manner similar to Figure 1, for the 12-day period following the shift to Run 3 nonreward in the $\mathbf{R}-\mathbf{N}-\mathbf{R}$ trial.

Separate analyses were done on each of the three runs, but a trials $\times$ days interaction appeared only on Run 3 $[F(11,66)=2.05, p<.05]$. There was no evidence of contrast effects, that is, slower running on $\mathrm{R}-\mathrm{N}-\mathrm{R}(\mathrm{N})$ than on R-N-N trials, in either Run 2 or Run 3. Since successive contrast may be interpreted as the consequence of unmet anticipations, we might expect to see contrast in animals anticipating reward yet experiencing nonreward, but contrast was not shown here in this rather unusual contrast design with sucrose. It remains to be seen whether the effect would appear in a comparable design with ordinary food pellets or with sucrose during extensive train- ing. These difficulties notwithstanding, the results of this experiment make prudent consideration of the possibility that the processes underlying interevent anticipation and successive contrast are different.

\section{REFERENCES}

AMSEL, A. (1958). The role of frustrative nonreward in noncontinuous reward situations. Psychological Bulletin, 55, 102-119.

BURNs, R. A. (1984). The goal units dimension in negative contrast failures with sucrose. Journal of General Psychology, 111, 9-23.

Burns, R. A., Lorig, T. S., CARR, E. J., \& WorThY, L. G. (1984). Alternation and reduction of liquid sucrose in discrete goal units. Journal of General Psychology, 111, 69-78.

Capaldi, E. J., NaWrocki, T. M., \& Verry, D. R. (1983). The nature of anticipation: An inter- and intraevent process. Animal Learning \& Behavior, 11, 193-198.

CAPALDI, E. J., \& VeRRY, D. R. (1981). Serial order anticipation learning in rats: Memory for multiple hedonic events and their order. Animal Learning \& Behavior, 9, 441-453.

ElliotT, M. H. (1928). The effect of change of reward on the maze performance of rats. University of California Publications in Psychology, 4, 19-30.

FlaherTy, C. F. (1982). Incentive contrast: A review of behavioral changes following shifts in reward. Animal Learning \& Behavior, 10, 409-440.

SELF, R., \& GAFFAN, E. A. (1983). An analysis of serial pattern learning by rats. Animal Learning \& Behavior, 11, 10-18.

SPENCE, K. W. (1956). Behavior theory and conditioning. New Haven, CT: Yale University Press.

Stanton, M., \& AMSEL, A. (1980). Adjustment to reward reduction (but not negative contrast) in rats 11,14 , and 16 days of age. Journal of Comparative and Physiological Psychology, 94, 446-458.

(Manuscript received for publication June 22, 1984.) 